\title{
Uma Ferramenta de Modelagem para Análise e Avaliação de Confiabilidade e Interoperabilidade em Sistemas-de-Sistemas por Meio de Heurísticas
}

\author{
Marcio Imamura, Francisco Henrique Cerdeira Ferreira (colaborador), \\ Rodrigo Pereira dos Santos (orientador) \\ ${ }^{1}$ Programa de Pós-Graduação em Informática \\ Universidade Federal do Estado do Rio de Janeiro (UNIRIO)
}

marcio.imamura@edu.unirio.br, ffrancisco.ferreira, rps\}@uniriotec.br

\begin{abstract}
The modern world is pushing organizations to innovate in the marketplace through increasingly agile, efficient, and cost-effective technology solutions. This scenario is leading system engineers to adopt solutions in the form of systems-of-systems (SoS), based on the integration of existing systems, instead of building new systems from scratch. Despite the benefits, there are concerns related to the reliability of $S o S$, since its constituent systems are independent. In this context, this research project aims to develop a modeling tool for SoS, focusing on their reliability and interoperability features. The expected impacts of this work are to facilitate communication between stakeholders of a SoS, as well as to provide more accurate analyzes of issues related to reliability and interoperability based on heuristics for engineering SoS.
\end{abstract}

Resumo. $O$ mundo moderno está forçando as organizações a se manterem competitivas no mercado por meio de soluções tecnológicas cada vez mais ágeis, eficientes e econômicas. Esse cenário está levando engenheiros de sistemas a adotarem soluções na forma de sistemas-de-sistemas (SoS), baseados na integração de sistemas existentes, ao invés de construir novos sistemas a partir do zero. Apesar dos benefícios, existem preocupações relacionadas à confiabilidade de um SoS, uma vez que os sistemas constituintes são independentes. Neste contexto, este projeto de pesquisa tem como objetivo desenvolver uma ferramenta de modelagem para SoS com foco em suas características de confiabilidade e interoperabilidade. Os impactos esperados deste trabalho são facilitar a comunicação entre os stakeholders, bem como prover análises mais precisas das questões relativas à confiabilidade e interoperabilidade com base em heurísticas para engenharia de SoS.

\section{Introdução}

Sistemas-de-Sistemas (SoS) são arranjos de sistemas independentes que colaboram entre si para cumprir uma missão, a qual não seria possível realizar por um sistema de forma isolada. Os SoS são caracterizados por seus constituintes terem (i) independência operacional, (ii) independência gerencial, (iii) distribuição geográfica, (iv) desenvolvimento evolucionário e (v) comportamento emergente resultante da colaboração sinérgica entre eles [Maier 1998]. Um exemplo notável de SoS são cidades inteligentes 
[Boscarioli et al. 2017], onde organizações trabalham de forma coordenada com seus sistemas de informação (SI) nas áreas da saúde, segurança e outros serviços públicos, a fim de melhorar a qualidade de vida dos cidadãos.

Nos últimos anos, o tema ganhou notoriedade tanto na indústria como na academia pelas oportunidades que foram criadas com a abordagem de SoS, que tem sido aplicada em diversos domínios, como segurança, saúde, mobilidade etc. [De Laurentis et al. 2007, Axelsson e Nylander 2018]. Ainda assim, existem diversos desafios relacionados à Engenharia de SoS, em especial, os relacionados com a garantia de qualidade. Embora tais temas sejam reconhecidamente importantes, há uma carência de soluções, principalmente no que diz respeito à modelagem de $\mathrm{SoS}$ para análise de atributos de qualidade, como confiabilidade e interoperabilidade.

Este estudo aborda dois atributos de qualidade no contexto de SoS: interoperabilidade, que diz respeito a como os sistemas constituintes trabalham de forma sinérgica visando o cumprimento dos objetivos do SoS e confiabilidade, que busca assegurar que um SoS opere como desejado, livre de falhas. A proposta do trabalho para a solução deste problema é: (i) identificar um conjunto de heurísticas para engenharia de SoS na literatura e (ii) construir uma ferramenta para avaliação de atributos de qualidade em SoS para tomada de decisão.

O restante deste artigo está organizado da seguinte forma: a Seção 2 apresenta o problema; na Seção 3, é descrita a solução proposta e a metodologia proposta para o trabalho; na Seção 4, é apresentado o projeto de avaliação da solução; e a Seção 5 traz as considerações finais.

\section{Apresentação do problema}

Existem diversas abordagens para o desenvolvimento e sustentação de SI tradicionais, que capazes de auxiliar em praticamente todas as tarefas relacionadas à construção de sistemas. Tais abordagens são utilizadas para diversos tipos de sistemas com características distintas como tamanho, complexidade, área de atuação, missão crítica etc. No entanto, as abordagens já estabelecidas para a engenharia de software muitas vezes não são aplicáveis ao contexto de SoS. Sendo assim, a engenharia de SoS vem sendo desenvolvida em paralelo com a engenharia de sistemas tradicionais [Bukowski 2016].

A engenharia de SoS é uma disciplina relativamente nova e, como tal, ainda carece de um ferramental adequado que facilite a modelagem e a avaliação de atributos de qualidade. A modelagem permite que os vários envolvidos como gestores, gerentes de projeto e desenvolvedores tenham a compreensão correta, em tempo de projeto, da arquitetura do sistema e de suas características de confiabilidade e a interoperabilidade. Além disso, a modelagem possibilita também uma análise do projeto, detectando problemas e oportunidades de otimização no funcionamento do SoS durante o seu ciclo de vida.

\section{Proposta de solução}

Serão realizados trabalhos em duas etapas: (i) identificação um conjunto de heurísticas adequadas a projetos de SoS capazes de nortear decisões em tempo de projeto e avaliar, de maneira mais objetiva, suas condições de funcionamento com foco em confiabilidade e interoperabilidade e (ii) construção de uma ferramenta de avaliação de atributos de qualidade em SoS, que considere a dinamicidade de sua arquitetura e que sirva também como 
instrumento de avaliação e tomada de decisão, comunicação entre os interessados no projeto, detecção de falhas e otimização da operação de SoS.

A ferramenta permitirá que as entidades de um SoS, bem como seus relacionamentos, possam ser representados graficamente utilizando como base o modelo conceitual proposto por Gonçalves et al. (2014) com o objetivo de permitir um entendimento consensual em SoS intensivos em software ( $\mathrm{SiSoS}$, do inglês Software-intensive SoS), isto é, uma classe de SoS. O projeto inicial da interface da ferramenta consiste em uma paleta de elementos que podem ser selecionados e conectados obedecendo a restrições de acordo com os tipos desses elementos e a funcionalidade esperada. Por exemplo, uma missão não pode deixar de estar conectada a elementos que forneçam algum tipo de capacidade para realizá-la. A Figura 1 representa, de maneira simplificada, a proposta inicial do funcionamento da ferramenta com notação e elementos fitícios, a atuação de um catálogo de heurísticas a serem selecionadas e a análise das características de qualidade.

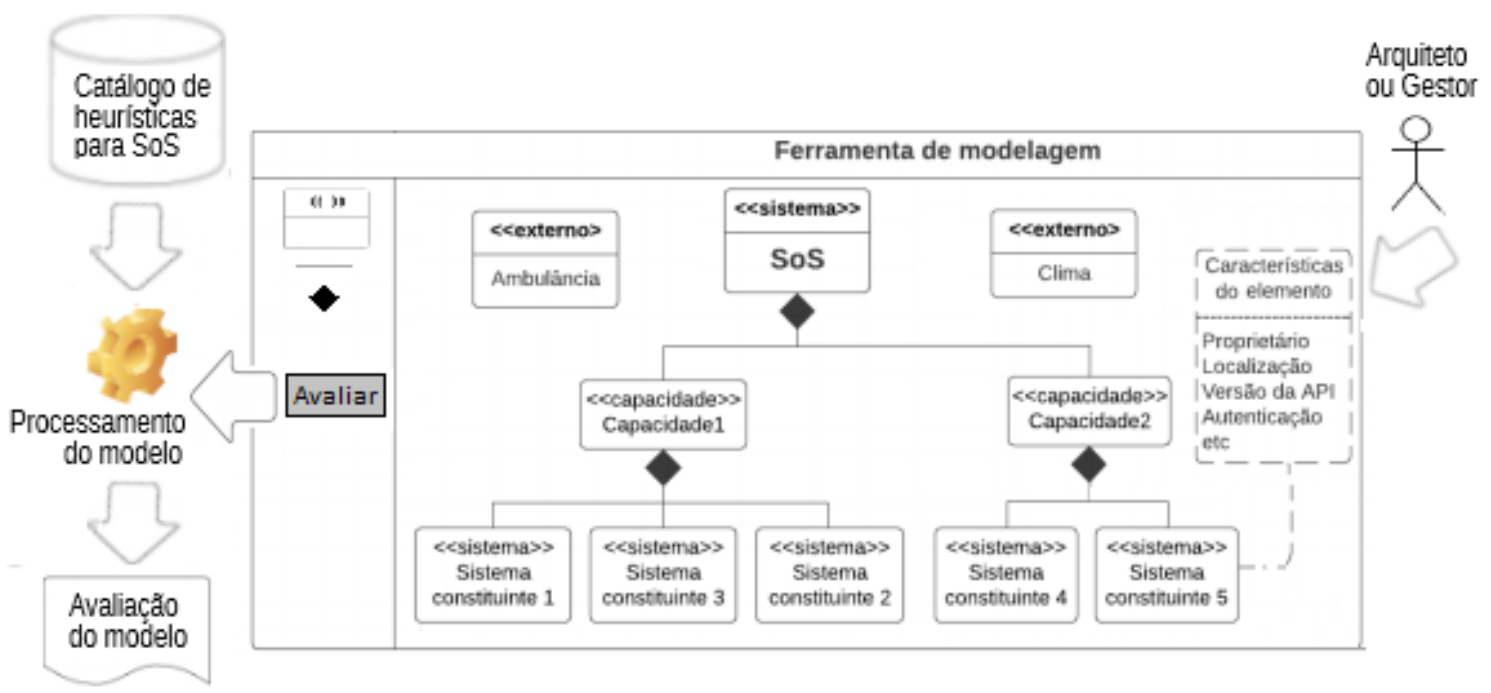

Figura 1. Esquema simplificado da ferramenta de modelagem proposta.

As heurísticas serão aplicadas, por exemplo, a entidades do modelo do SoS ligadas a questões como interfaces e redundâncias. As sugestões e restrições oriundas das heurísticas serão aplicadas durante a modelagem, facilitando a interlocução entre os envolvidos na construção do SoS. Isto permitirá aos engenheiros do SoS terem à sua disposição funcionalidades que permitam utilizar o modelo e a aplicação das heurísticas para identificar problemas de projeto e oportunidades de melhorias da arquitetura, interfaces e redundâncias do SoS. A ferramenta deve incorporar as heurísticas identificadas na etapa (i) a fim de auxiliar gestores e engenheiros de SoS na tomada de decisão.

\subsection{Metodologia}

As atividades propostas são nesse estudo são: (i) por meio de um estudo utilizando mapeamentos sistemáticos e revisões sistemáricas da literatura, identificar como as características de confiabilidade e de interoperabilidade, assim como as heurísticas envolvidas na formação e operação de SoS, estão sendo tratadas e selecionar quais podem ser incorporadas na ferramenta de modelagem e identificar profissionais com conhecimento tácito 
em construção de SoS para verificar os resultados obtidos; (ii) condução do projeto de construção da ferramenta para avaliação de SoS visando representar graficamente a sua arquitetura e as características encontradas nos estudos conduzidos; e (iii) avaliar a ferramenta produzida com profissionais a fim de verificar a sua adequação a projetos em ambiente de produção.

\section{Projeto de avaliação da solução}

As heurísticas para construção e funcionamento do SoS, a ferramenta de modelagem e avaliação do SoS, assim como as características de confiabilidade e interoperabilidade, serão avaliadas com especialistas da área por meio de entrevistas e utilização da ferramenta, demostrando também a tecnologia utilizada, as referências empregadas e o processos para avaliação do modelo construído. Estes especialistas serão selecionados na indústria e na academia.

\section{Considerações finais}

Ainda que a pesquisa em SoS tenha avançado nos últimos anos, ainda existem barreiras a serem superadas. Entre os desafios, estão os relacionadas à modelagem e análise de atributos de qualidade desses sistemas. Assim, a contribuição deste trabalho consiste em desenvolver um catálogo de heurísticas que sejam adequadas para projetar SoS e capazes de tratar problemas de confiabilidade e interoperabilidade nesse tipo de sistemas. Esse catálogo de heurísticas será incorporado a uma ferramenta de avaliação para tratar questões de qualidade em SoS com foco em confiabilidade e interoperabilidade. Isso servirá para que projetistas consigam, por meio do modelo e da aplicação das heurísticas sobre esse modelo, identificar problemas ou oportunidades de melhorias no SoS, além de auxiliar nas tomadas de decisão sobre aspectos técnicos como arquitetura, interfaces e redundância.

\section{Referências}

Axelsson, J. e Nylander, S. (2018). An analysis of systems-of-systems opportunities and challenges related to mobility in smart cities. In 2018 13th Annual Conference on System of Systems Engineering (SoSE), pages 132-137. IEEE.

Boscarioli, C., Araujo, R. M., e Maciel, R. S. P. (2017). I GranDSI-BR - Grand Research Challenges in Information Systems in Brazil 2016-2026. Special Committee on Information Systems (CE-SI). Brazilian Computer Society (SBC). 184p.

Bukowski, L. (2016). System of systems dependability-theoretical models and applications examples. Reliability Engineering \& System Safety, 151:76-92.

De Laurentis, D., Dickerson, C., DiMario, M., Gartz, P., Jamshidi, M. M., Nahavandi, S., Sage, A. P., Sloane, E. B., e Walker, D. R. (2007). A case for an international consortium on system-of-systems engineering. IEEE Systems Journal, 1(1):68-73.

Gonçalves, M. B., Cavalcante, E., Batista, T., Oquendo, F., e Nakagawa, E. Y. (2014). Towards a conceptual model for software-intensive system-of-systems. In 2014 IEEE International Conference on Systems, Man, and Cybernetics (SMC), pages 1605-1610. IEEE.

Maier, M. W. (1998). Architecting principles for systems-of-systems. Systems Engineering: The Journal of the International Council on Systems Engineering, 1(4):267-284. 